\title{
Reliability-based optimization of maintenance scheduling of mechanical components with the method of accelerated tests
}

\author{
Case of a parallel series system
}

\author{
CHOUAIRI Asmâa, EL GHORBA Mohamed, \\ BENALI Abdelkader \\ Laboratory of Control and Mechanical Characterization of \\ Materials and Structures (LCCMMS), ENSEM, BP 8118 \\ Oasis, Hassan II University, \\ Casablanca (Morocco) \\ Chouairi.asmaa@gmail.com
}

\author{
HACHIM Abdelilah \\ Laboratory of Mechanics, Faculty of Science Ain Chock \\ (FSAC), BP 5366 Maarif, Hassan II University, \\ Casablanca, Morocco
}

\begin{abstract}
To have effective production planning and control, it is necessary to calculate the reliability and maintenance of a production system as a whole. Therefore, with today's highly reliable components, we are often unable to obtain a reasonable amount of test data under normal use condition. For this reason, accelerated tests method is the reasonable procedure to be applied. It is used to determine the reliability of a product in a short period of time by accelerating the use environment. Application of the method of accelerated tests can save cost that we used to pay for overcoming the dilemma of not being able to estimate failure rates by testing directly at use conditions.

In this paper, we propose a practical method for system reliability analysis. Among the existing methods for system reliability analysis, reliability graph theory is particularly attractive due to its intuitiveness, which is an extension of the conventional reliability graph. A function library was developed and designed to calculate the reliability-maintainability and availability of parallel series complex systems, whose functional description is translated into a block diagram that combines in series and parallel components studied. An Extensive analytical modeling study has been performed also to verify the effectiveness of the proposed technique.
\end{abstract}

Keywords-reliability; maintenance; method of accelerated tests; failure rates; availability; parallel series system.

\section{INTRODUCTION}

The operation of industrial utility systems offer multiple degrees of freedom (e.g. equipment sizes, number of units, and their loads) that can be exploited to achieve large economic savings.

At the same time, since no equipment is one hundred percent reliable, it is necessary to account for the possibility of failure together with preventive and corrective maintenance periods of all the items of the plant.

In this way, reliability and availability issues not only have a major impact on the design and operation of a utility system, but also they considerably increase the number of options that should be assessed to reduce capital and/or operating costs.
Hence, minimizing such expenditure represents a very challenging task due to the highly combinatorial computations involved and strong interrelations between the equipment. This means that the whole utility system must be simulated in order to take into account all the potential units and determine the plant-wide consequences of any proposed modification.

Moreover, only with this approach it is possible to assess which design and/or operational decisions actually improves the overall economics of the system.

Generally, Reliability can be defined [1] as the probability that a device or system will perform a required function at a given point in time, when operated under specific conditions. In other words, reliability is a quantitative measure of nonfailure operation over an (operational) time interval. It is important to note that this definition assumes that certain criteria have been previously established to clearly specify what is considered to be the intended function of the item.

In addition, reliability should be also specified for a given period of time as this variable also has an important effect. For instance, a compressor becomes less reliable as its number of operating hours (without being switched off for maintenance) increases.

In addition, failure normally implies a corrective maintenance action corresponding to the repair time needed to bring an element back to regular operation. Also, the equipment often requires preventive or scheduled maintenance to improve its reliability. Hence, the down time of a unit is comprised of both its corrective and preventive maintenance periods. Thereafter, availability is defined as the probability that an item performs its required function during a certain interval of time (including maintenance periods) whilst operated under specific conditions. In other words, it is the percentage the item is operating over a specified time interval encompassing also its maintenance periods.

It is worth mentioning the concept of maintainability which might correspond to the probability of completing a repair action during a certain time interval [2] [3], or it can 
denote the probability of performing preventive maintenance during a certain time interval [4].

In any case, it must not be confused with the downtime or with the complement of availability. Thus, while reliability is a measure of non-failure operation of an item, maintainability is related to its capability of being repaired or to its need of receiving maintenance. Thereafter, both measures can be used to calculate the overall availability, representing the net operational time of an element or system.

In this paper, we present two methods, the reliabilityclassical method and the method of accelerated tests [5], implemented by a graphical calculation developed by numerical approach for a parallel series system. We illustrate and compare these two approaches by using the rough estimate of gap that represents the gain in reliability between the two methods and estimating the relation reliabilitymaintainability and availability.

\section{RELIABILITY METHOD OF ACCELERATED TESTING}

The classical reliability theory determines how to evaluate a reliability function with the fraction of life appropriate to the system [6] [7].

Under accelerated tests have been developed many models statistics correlating the lifetimes of failure modes Nelson [8] has indicated that the stress can be applied in various ways, commonly used methods are step-stress and constant- stress. Under step-stress, a test item is first run at normal use condition and, if it does not fail then, it is run at accelerated condition until failure occurs or the observation is censored. But the constant-stress runs each item either at normal use condition or at accelerated condition only. Accelerated test condition includes stress(s) in the form of temperature, voltage, pressure, vibration, cycling rate, humidity, etc.

DeGroot and Goel [9] have introduced the concept of accelerated tests in which a test item is first run at use condition and, if it does not fail for a specified time, then, it is run at accelerated condition until failure. This implies that the test is shifted to the higher level of stress and it is continued until the pre-specified condition, i.e., if the items have not failed by some prespecified time or number of failures. The effect of this shifting is to multiply the remaining lifetime of the item by an unknown factor which is known as acceleration factor.

Bhattacharyya and Soejoeti [10] have indicated that the method of accelerated tests is practical for many problems of life testing where the test process requires a long time if the test is simply carried out under the use condition.

The method of accelerated tests has been, considered by several authors. Bai and Chung [11] have used the maximum likelihood method to estimate the scale parameter and the acceleration factor for exponentially distributed life-time. Abdel- Ghally et al. [12] have used the maximum likelihood method to estimate the acceleration factor and the parameters of Weibull distribution. Abdel-Ghani [13] considered the estimation problem of log-logistic distribution parameters under step-stress. Bai et al. [14] used the maximum likelihood method to estimate the scale parameter and the acceleration factor for the log normally distributed lifetime.

Meng [15] compared the MTTF of four series-parallel and parallel-series redundant systems which contained $2 \mathrm{n}$ independent components. General ordering relations between four different systems arising in standby redundancy enhancement in terms of their MTTF are proposed by Meng [16]. Lewis [17] first introduced the concept of the standby switching failures in the reliability with standby system. Kuo and Zuo [18] introduced many of the system reliability models such as parallel, series, standby, multistate, maintainable system, etc. Gaikowsky et al. [19] and Wang and Pearn [20] examined the series systems with cold standby components and warm standby components, respectively. Wang and Kuo [21] investigated the cost and probabilistic analysis of series systems with mixed standby components.

Wang and Ke [22] studied the probability analysis of a repairable system with warm standbys plus balking and reneging for which no cost-benefit analysis is considered. Recently, Wang et al. [23] proposed cost-benefit analysis of series systems with warm standby components and general repair time. To the best of our knowledge, four different system configurations with warm standby components involving standby switching failures has never been investigated.

The problem considered in this paper is more general than the works of Sivazlian and Wang [24].

We first provide a systematic methodology to develop the explicit expressions reliability-maintainability and availability. Next, we use an efficient Matlab computer program to perform a parametric investigation. We provide extensive numerical computations to study the effects of various values of system parameters to the cost/benefit ratios. Finally, we rank four configurations for the MTTF and MTTR, based on specific values of distribution parameters, as well as of the costs of the component.

\section{PARALLEL SERIES SYSTEM}

\section{A. Relation between reliability-failure and fraction of life}

We consider a system of $n$ blocks characterized by the same reference law, but experiencing different levels of stress. In the case of a configuration composed by $S=5$ series blocks, each block is composed by $\mathrm{P}=5$ single elements connected in parallel where the reference law is a reliabilityseries law, we will have an expression of reliability as (1):

$R_{p s}=1-\left[\left(1-\exp \left(-S . \beta^{\lambda}\right)\right)^{p}\right]^{\sum_{i=1}^{n}\left(A_{F i}\right)^{\lambda}}$

$\mathrm{R}_{\mathrm{ps}}$ : Reliability of parallel series system

$\mathrm{S}$ : number of components in series

$\mathrm{P}$ : number of components in parallel

$\beta$ : form parameter

$\gamma$ : position or origin parameter

$\mathrm{A}_{\mathrm{F}}$ : Factor of accelerated method 
The major difference with the classical law of reliability is that the reliability function depends on maintenance factor through the parameter $\mathrm{A}_{\mathrm{F}}$.

The failure of a series system is based on the fraction of life, and given by (2):

$Q_{p s}=\left[\left(1-\exp \left(-S \cdot \beta^{\lambda}\right)\right)^{p}\right]^{\sum_{i=1}^{n}\left(A_{F i}\right)^{\lambda}}$

$\mathrm{Q}_{\mathrm{ps}}$ : Failure of parallel series system

S: number of components in series

$\mathrm{P}$ : number of components in parallel

$\beta$ : form parameter

$\gamma$ : position or origin parameter

$\mathrm{A}_{\mathrm{F}}$ : Factor of accelerated method

\section{B. Reliability between reliability and damage}

Concerning the mechanical damage model that reflects the behavior of the component toward the failure mode that is the fatigue, is based on the reduction of strength and endurance limit, these features are results of the different damage that the metal may has, this model is the unified theory which was established after a thorough overview of the different theories that describe the damage in the solicitation of fatigue.

The expression (3) describes the unified theory based on the fraction of life $\beta$ :

$$
D=\frac{\beta}{\beta+(1-\beta)\left(\frac{\gamma-\left(\frac{\gamma}{\gamma_{u}}\right)^{m}}{\gamma-1}\right)}
$$

With $\gamma=\Delta \sigma / \sigma_{0}$ and $\gamma \mathrm{u}=\sigma_{\mathrm{u}} / \sigma_{0}$ where $\Delta \sigma$ is the amplitude of solicitation, $\sigma_{0}$ the endurance limit of virgin material, $\sigma_{\mathrm{u}}$ the ultimate stress of virgin material and $\mathrm{m}$ is a parameter related to the material $(\mathrm{m}=8$ for steel). We set (4):

$$
\alpha=\left(\frac{\gamma-\left(\frac{\gamma}{\gamma_{u}}\right)^{8}}{\gamma-1}\right)
$$

This gives (5):

$$
D=\frac{\beta}{\beta+(1-\beta) \cdot \alpha}
$$

So, we introduce the damage variable in the model of reliability, by expressing $\mathrm{D}$, as a function of fraction of life to connect reliability to damage, resulting in (6):

$$
\beta=\frac{\alpha \cdot D}{1-D(1-\alpha)}
$$

According to the equation for series system reliability, we deduce (7):

$\left\{\begin{array}{l}R_{p s}=1-\left[\left[1-\exp \left(-S \cdot\left(\frac{\alpha \cdot D}{1-D(1-\alpha)}\right)^{\lambda}\right)\right]^{p}\right]^{\sum_{i=1}^{n}\left(A_{F i}\right)^{\lambda}} \\ 1 / e \leq R \prec 1 \\ D=0 \text { pour } R=1\end{array}\right.$

The failure of a series system based on the damage is given by the relation (8):

$Q_{p s}=\left[\left[1-\exp \left(-S \cdot\left(\frac{\alpha . D}{1-D(1-\alpha)}\right)^{\lambda}\right)\right]^{p}\right]^{\sum_{i=1}^{n}\left(A_{F i}\right)^{\lambda}}$

For: $\gamma=\Delta \sigma / \sigma_{0}=(1.2) ; \lambda=(1.5) ; \gamma_{\mathrm{u}}=\sigma_{\mathrm{u}} / \sigma_{0}=2 ; \mathrm{S}=5$ components; $\mathrm{P}=5$ components.

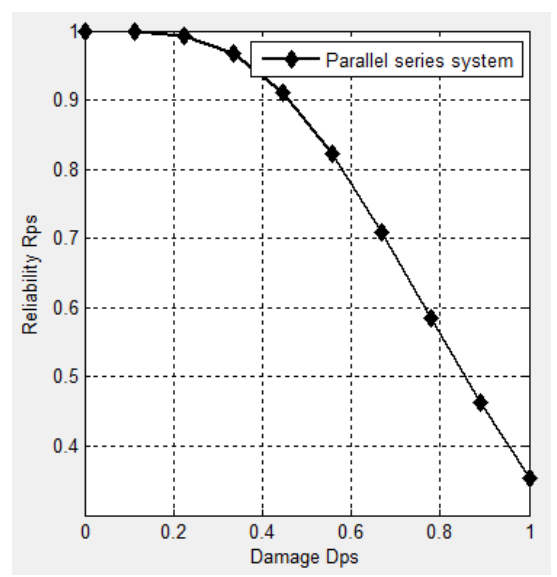

Fig. 1. Reliability in terms of damage for a parallel series system

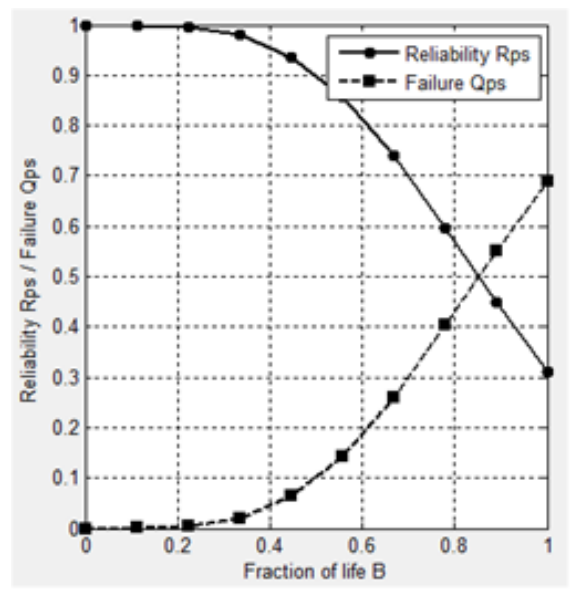

Fig. 2. Superposition of the curves of reliability and failure for a parallel series system 
We keep the same number of series blocks and the number of parallel components (Fig. 1 and Fig. 2).

The influence of the parallel trend of the components on the system reliability appears so clear. The opposite is observed: the system is less reliable if we raise the number of series blocks, and we keep the same number of parallel components.

\section{Relation relibaility-maintainability}

The Mean time between failures (MTBF) and the Mean time to recovery (MTTR) are given by relations (9) and (10).

$$
\begin{aligned}
& \operatorname{MTBF}_{p s}=\int_{0}^{1} 1-\left[\left(1-\exp \left(-S . \beta^{\lambda}\right)\right)^{p}\right]_{i=1}^{n}\left(\frac{t_{r e f}}{t_{i}-\gamma}\right)^{\lambda} d t \\
& \operatorname{MTTR}_{p s}=(M T B F)_{p s}^{-1}
\end{aligned}
$$

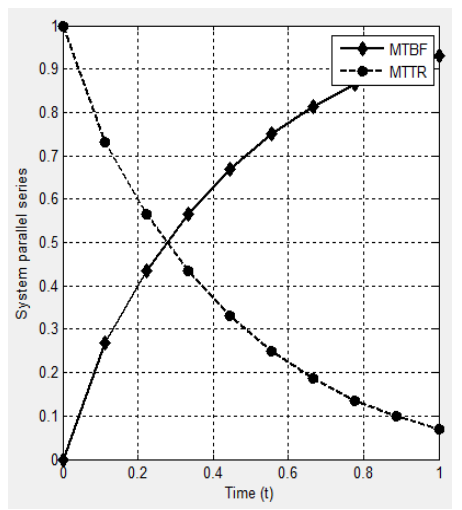

Fig. 3. Relation reliability-maintainability for a parallel series system

In fig. 3 the superposition of curves of MTBF and MTTR shows a reversal of situation at intersection of the two curves. The system reached set point at a fraction of $50 \%$ with a time of $28 \%$. This approach allowed us to predict the critical moment of the damage, and thus to intervene in a suitable time to maintain the system.

\section{Availability}

For a parallel series system availability is given by (11).

$D_{p s}=\frac{M T B F_{p s}}{M T T R_{p s}+M T B F_{p s}}$

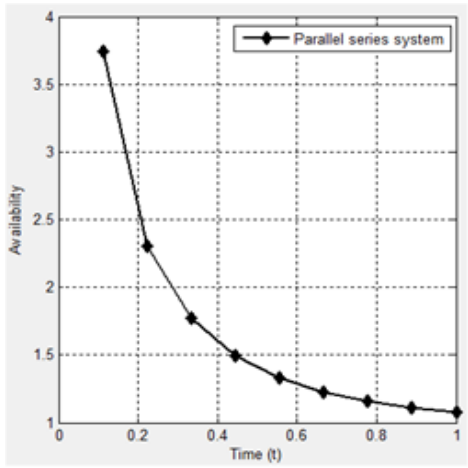

Fig. 4. Availability in function of time for a parallel series system
According to Fig. 4, we deduce clearly that there is a rapid decrease in availability; this decrease is increasingly proportional to the function of time.

\section{COMPARISON OF CONVENTIONAL AND RELIABILITY- MODEL ACCELERATED TESTS}

\section{A. Numerical analysis}

In this part we use a library to calculate the reliability of complex systems, that is to say systems whose functional description can be translated into a block diagram that combines, in series or in parallel, components (or failure modes) for which the law of reliability must be known a priori. The characteristics of the components (the law of reliability, hours of work already accomplished, the control component), are saved in a library (fig. 5).

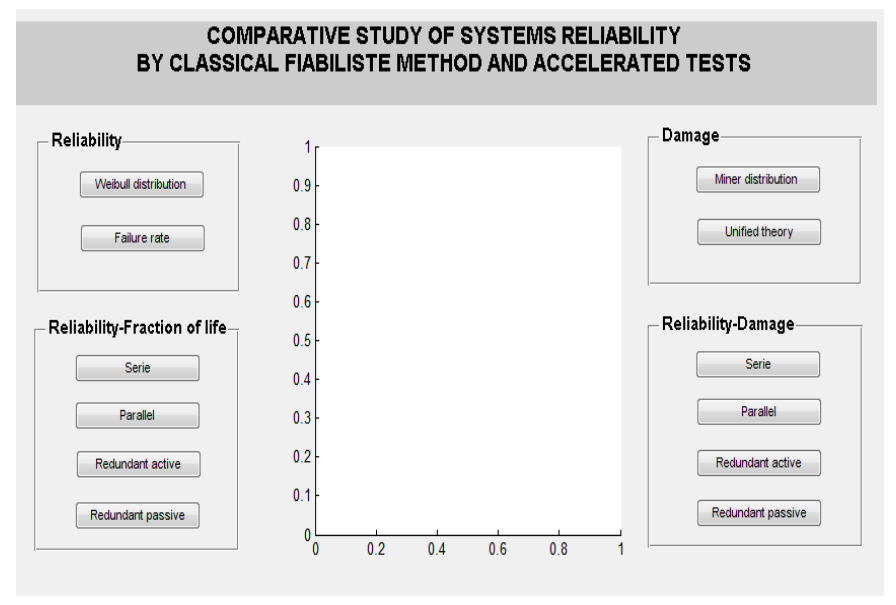

Fig.5. Interface library for comparative study of reliability systems

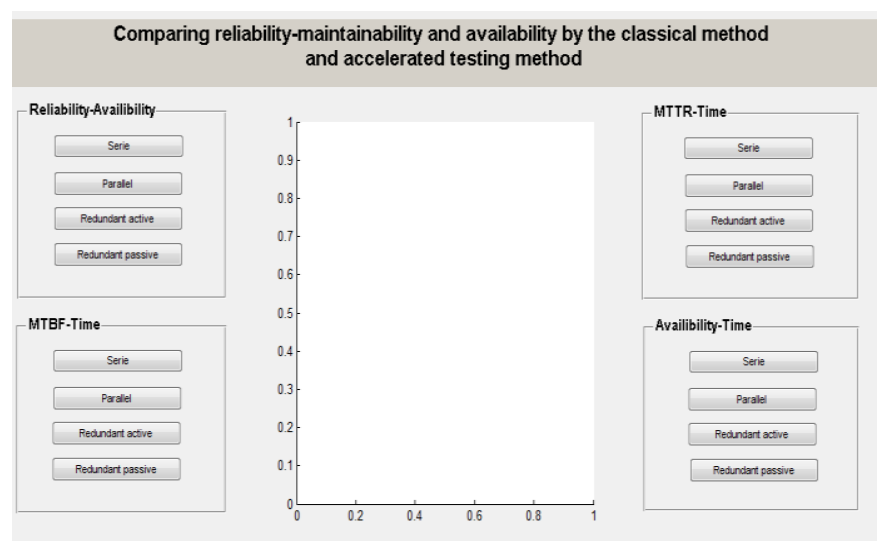

Fig. 6. Interface library for comparative study of reliability-maintainability and availability

To treat the model, we use an algorithm derived from graph theory, which generates digitally function of system reliability.

The interface module to load and present the results graphically is shown in fig. 6; the reliability function can be easily calculated classical compared with the reliability of 
accelerated testing, facilitating the search for greater reliability.

\section{B. Weibull distribution}

This is a law that can be adjusted correctly to all kinds of experimental results and operational cleverly choosing the parameter values. As it covers the case where the failure rate is variable and can therefore be easily adapted to periods of youth and various forms of aging systems

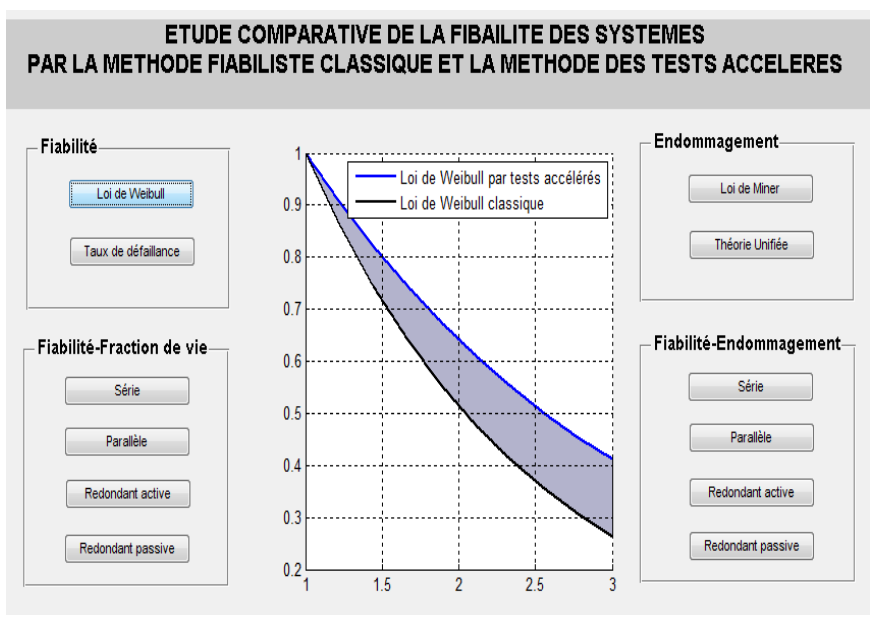

Fig. 7. Comparison of Weibull distribution between the two methods

The fig. 7 shows a comparison of the Weibull distribution for reliability classical method and accelerated tests. There is a proportional improvement of reliability caused by the reliability of the accelerated testing method, which is about $15 \%$ to end of the observation period. The difference between the two methods is the gain of reliability to quantify the benefits (cost, quality) of a replacement or, similarly, any editing operation.

The reliability of the accelerated testing method represents an adjustment to the traditional reliability law, and it will allow a timely intervention for preventive maintenance of the system.

\section{Parallel series system}

For $=\Delta \sigma / \sigma_{0}=(1.2) ; \quad \lambda=(1.5) ; \quad \gamma_{\mathrm{u}}=\sigma_{\mathrm{u}} / \sigma_{0}=2 ; \mathrm{S}=5$ components; $\mathrm{P}=5$ components.

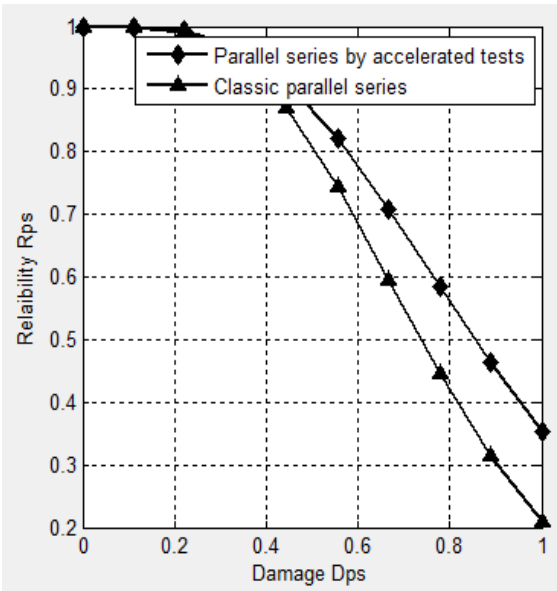

Fig. 8 . Comparison of the reliability depending on damage for a parallel series system

We deduce clearly in fig. 8 that there is a rapid decrease of reliability for the two curves, and this decrease is greater for the reliability in accelerated testing, and thus leads to a gain of reliability and internal resistance component of $16 \%$, which will keep longer system operation in service.

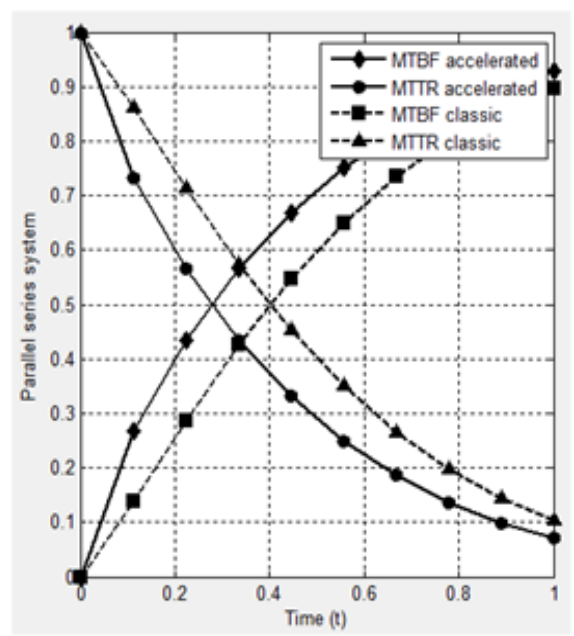

Fig. 9. Superposition of curves of reliability and for a parallel series system

The superposition between curves of reliability and damage in fig. 9 diverges and has no point of intersection for classical reliability method. The method of accelerated testing shows an intersection that requires a critical maintenance to 82 $\%$ fraction of the system life. 


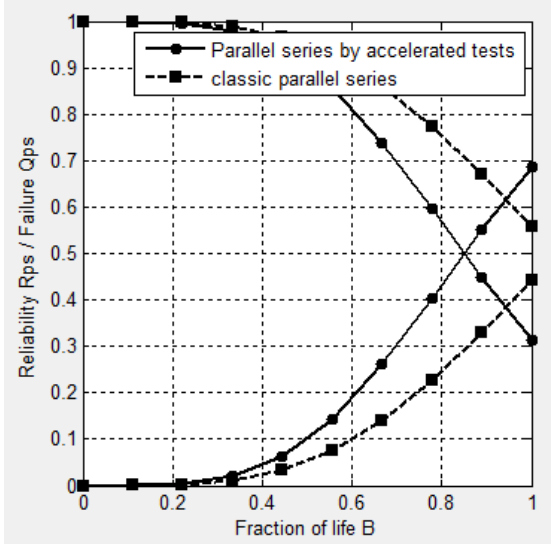

Fig 10. Superposition of curves of reliability-availability for parallel series system

The fig. 10 shows the intersection curves of the MTBF and MTTR by the classical method and the method of accelerated tests for a parallel series system. We deduce that the intersection curves of the conventional method is carried out at $40 \%$ fraction of the time and $50 \%$ of the total fraction, also the intersection curves of the method of accelerated testing is performed at $28 \%$ of the fraction of time and $50 \%$ of the total fraction, which requires an immediate and precise to avoid failures.

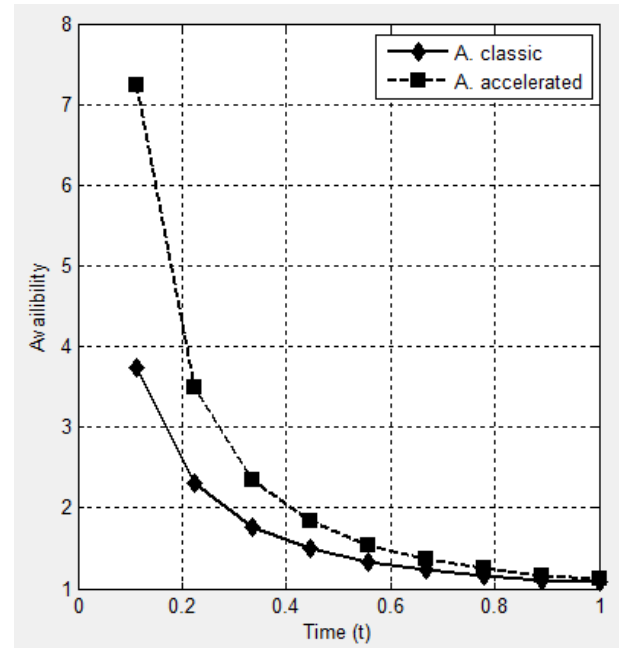

Fig. 11. Superposition of curves of reliability-availability for parallel series system

The fig. 11 shows the intersection curves of availability by the conventional method and the method of accelerated tests. We deduce that the availability given by the theory of accelerated tests reached a large value at the beginning of operation compared to the conventional method. This reflects that the accelerated method is more efficient and accurate.

\section{CONCLUSION}

Regarding availability and reliability issues, due to the complexities of handling these concerns within a systematic methodology, in most cases the redundant elements of a utility plant are pre-specified according to heuristics or rules of thumb.

Our goal is to determine the reliability of complex multi component services. The theory enables accelerated testing, initially, to determine the reliability of system components through the acceleration factor $\mathrm{A}_{\mathrm{F}}$. This parameter depends on the positional parameter Weibull, failure mode and associated stress.

With the increasing complexity of systems that results in costs of operation and maintenance costs. We therefore sought to reduce maintenance and improve reliability by using numerical analysis that allowed us to illustrate a comparison based on two methods. The results show that the reliability of the accelerated testing method represents an adjustment to traditional reliability law, so it can provide support predictive maintenance for the determination of optimal periods between interventions and quantify the benefits of reliability as a result of replacement or modification.

\section{REFERENCES}

[1] Ebeling, C., 1997. An Introduction to Reliability and Maintainability Engineering. McGraw-Hill, New York.

[2] Govil, A.K., 1983. Reliability Engineering. McGraw-Hill, New Delhi.

[3] Olsommer, B., Favrat, D., Spakovsky, M.R., 1999a. An approach for the time-dependent thermoeconomic modeling and optimization of energy system synthesis, and operation---part 1. International Journal of Applied Thermodynamics, 2 (3), 97--113.

[4] Olsommer B., Favrat D., Spakovsky M.R., 1999b. An approach for the time-dependent thermoeconomic modeling and optimization of energy system synthesis, and operation---part 2. International Journal of Applied Thermodynamics, 2 (4), 77--186.

[5] F. Guerin, O.Tebbi and B.Dumon, B. Accelerated testing based on mechanical damage model. In IEEE International Conference on Reliability and Maintenability, Philadelphia, Pennsylvania, USA.2001.

[6] M.Chahid . Optimization of the reliability-fatigue damage of a composite glass fiber-polyepoxide. University Hassan 2 Mohammedia, Ph.D. Thesis, 2003.

[7] A. Maziri. Optimization of the fatigue damage of metallic materials. Memory of DESA, ENSEM, Casablanca, November 2003.

[8] W. Nelson, Accelerated Testing: Statistical Models, Test Plans, and Data Analysis, John Wiley \& Sons, New York, 1990.

[9] M.H. DeGroot, P.K. Goel, Bayesian estimation and optimal designs in partially accelerated life testing, Nav. Res. Logist. Quart. 26 (1979) 223-235.

[10] G.K. Bhattacharyya, Z. Soejoeti, A tampered failure rate model for stepstress accelerated life test, Commun. Stat. - Theory Method 18 (1989) 16271643.

[11] D.S. Bai, S.W. Chung, Optimal design of partially accelerated life tests for the exponential distribution under Type-I censoring, IEEE Trans. Reliab. 41 (1992) 400-406.

[12] A.A. Abdel-Ghally, A.F. Attia, M.M. Abdel-Ghani, The maximum likelihood estimates in step partially accelerated life tests for the Weibull parameters in censored data, Commun. Stat. - Theory Method 31 (2002) 551573.

[13] M.M. Abdel-Ghani, The estimation problem of the log logistic parameters in step partially accelerated life tests using Type-I censored data, Natl. Rev. Social Sci. 41 (2004) 1-19.

[14] D.S. Bai, S.W. Chung, Chun YR, Optimal design of partially accelerated life tests for lognormal distribution under Type-I censoring, Reliab. Eng. Syst. Safe. 40 (1993) 85-92. 
[15] F.C. Meng, On comparison of MTBF between four redundant systems, Microelectronics and Reliability 33 (1993) 1987-1990.

[16] F.C. Meng, Comparing the MTBF of four systems with standby components, Microelectronics and Reliability 35 (1995) 1031-1035.

[17] E.E. Lewis, Introduction to Reliability Engineering, second ed., John Wiley \& Sons, New York, 1996.

[18] W. Kuo, M.J. Zuo, Optimal Reliability Modeling: Principles and Applications, John Wiley \& Sons, New York, 2003.

[19] C. Galikowsky, B.D. Sivazlian, P. Chaovalitwongse, Optimal redundancies for reliability and availability of series systems, Microelectronics and Reliability 36 (1996) 1537-1546.

[20] K.-H. Wang, W.L. Pearn, Cost benefit analysis of series systems with warm standby components, Mathematical Methods of Operations Research 58 (2003) 247-258.
[21] K.-H. Wang, C.-C. Kuo, Cost and probabilistic analysis of series systems with mixed standby components, Applied Mathematical Modelling 24 (2000) 957-967.

[22] K.-H. Wang, J.-C. Ke, Probabilistic analysis of a repairable system with warm standbys plus balking and reneging, Applied Mathematical Modelling 27 (2003) 327-336.

[23] K.-H. Wang, Y.-C. Liou, W.L. Pearn, Cost benefit analysis of series systems with warm standby components and general repair times, Mathematical Methods of Operations Research 61 (2005) 329-343.

[24] F.C. Meng, On comparison of MTBF between four redundant systems, Microelectronics and Reliability 33 (1993) 1987-1990.

\section{Creative Commons Attribution License 4.0} (Attribution 4.0 International, CC BY 4.0)

This article is published under the terms of the Creative Commons Attribution License 4.0

https://creativecommons.org/licenses/by/4.0/deed.en_US 